\title{
A Geometric Proof that $e$ is Irrational and a New Measure of its Irrationality
}

\section{Jonathan Sondow}

1. INTRODUCTION. While there exist geometric proofs of irrationality for $\sqrt{ } 2$ [2], [27], no such proof for $e, \pi$, or $\ln 2$ seems to be known. In section 2 we use a geometric construction to prove that $e$ is irrational. (For other proofs, see [1, pp. 27-28], [3, p. 352], [6], [10, pp. 78-79], [15, p. 301], [16], [17, p. 11], [19], [20], and [21, p. 302].) The proof leads in section 3 to a new measure of irrationality for $e$, that is, a lower bound on the distance from $e$ to a given rational number, as a function of its denominator. A connection with the greatest prime factor of a number is discussed in section 4. In section 5 we compare the new irrationality measure for $e$ with a known one, and state a numbertheoretic conjecture that implies the known measure is almost always stronger. The new measure is applied in section 6 to prove a special case of a result from [24], leading to another conjecture. Finally, in section 7 we recall a theorem of G. Cantor that can be proved by a similar construction.

2. PROOF. The irrationality of $e$ is a consequence of the following construction of a nested sequence of closed intervals $I_{n}$. Let $I_{1}=[2,3]$. Proceeding inductively, divide the interval $I_{n-1}$ into $n(\geq 2)$ equal subintervals, and let the second one be $I_{n}$ (see Figure 1). For example, $I_{2}=\left[\frac{5}{2}, \frac{6}{2 !}\right], I_{3}=\left[\frac{16}{3 !}, \frac{17}{3 !}\right]$, and $I_{4}=\left[\frac{65}{4 !}, \frac{66}{4 !}\right]$.

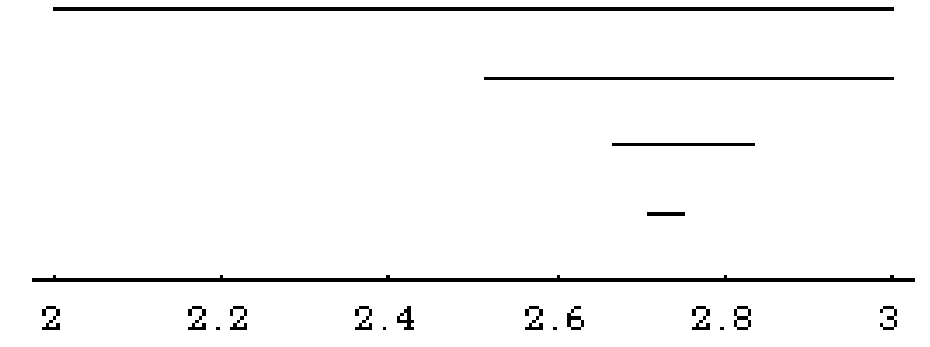

Figure 1. The intervals $I_{1}, I_{2}, I_{3}, I_{4}$.

The intersection

$$
\bigcap_{n=1}^{\infty} I_{n}=\{e\}
$$

is then the geometric equivalent of the summation (see the Addendum)

$$
\sum_{n=0}^{\infty} \frac{1}{n !}=e
$$


When $n>1$ the interval $I_{n+1}$ lies strictly between the endpoints of $I_{n}$, which are $\frac{a}{n !}$ and $\frac{a+1}{n !}$ for some integer $a=a(n)$. It follows that the point of intersection (1) is not a fraction with denominator $n$ ! for any $n \geq 1$. Since a rational number $p / q$ with $q>0$ can be written

$$
\frac{p}{q}=\frac{p \cdot(q-1) !}{q !}
$$

we conclude that $e$ is irrational.

Question. The nested intervals $I_{n}$ intersect in a number-let's call it $b$. It is seen by the Taylor series (2) for $e$ that $b=e$. Using only standard facts about the natural logarithm (including its definition as an integral), but not using any series representation for log, can one see directly from the given construction that $\log b=1$ ?

3. A NEW IRRATIONALITY MEASURE FOR $\boldsymbol{e}$. As a bonus, the proof leads to the following measure of irrationality for $e$.

Theorem 1. For all integers $p$ and $q$ with $q>1$

$$
\left|e-\frac{p}{q}\right|>\frac{1}{(S(q)+1) !}
$$

where $S(q)$ is the smallest positive integer such that $S(q)$ ! is a multiple of $q$.

For instance, $S(q)=q$ if $1 \leq q \leq 5$, while $S(6)=3$. In 1918 A. J. Kempner [13] used the prime factorization of $q$ to give the first algorithm for computing

$$
S(q)=\min \{k>0: q \mid k !\}
$$

(the so-called Smarandache function [28]). We do not use the algorithm in this note.

Proof of Theorem 1. For $n>1$ the left endpoint of $I_{n}$ is the closest fraction to $e$ with denominator not exceeding $n$ !. Since $e$ lies in the interior of the second subinterval of $I_{n}$,

$$
\left|e-\frac{m}{n !}\right|>\frac{1}{(n+1) !}
$$

for any integer $m$. Now given integers $p$ and $q$ with $q>1$, let $m=p \cdot S(q) ! / q$ and $n=S(q)$. In view of (5), $m$ and $n$ are integers. Moreover,

Therefore, (6) implies (4).

$$
\frac{p}{q}=\frac{p \cdot S(q) ! / q}{S(q) !}=\frac{m}{n !} .
$$


As an example, take $q$ to be a prime. Clearly, $S(q)=q$. In this case, (4) is the (very weak) inequality

$$
\left|e-\frac{p}{q}\right|>\frac{1}{(q+1) !} .
$$

In fact, (4) implies that (8) holds for any integer $q$ larger than 1, because $S(q) \leq q$ always holds. But (4) is an improvement of (8), just as (7) is a refinement of (3).

Theorem 1 would be false if we replaced the denominator on the right side of (4) with a smaller factorial. To see this, let $p / q$ be an endpoint of $I_{n}$, which has length $\frac{1}{n !}$. If we take $q=n !$, then since evidently

$$
S(n !)=n
$$

and $e$ lies in the interior of $I_{n}$,

$$
\left|e-\frac{p}{q}\right|<\frac{1}{S(q) !} .
$$

(If $q<n !$, then (10) still holds, since $n>2$, so $p / q$ is not an endpoint of $I_{n-1}$, hence $S(q)=n$.)

4. THE LARGEST PRIME FACTOR OF $q$. For $q \geq 2$ let $P(q)$ denote the largest prime factor of $q$. Note that $S(q) \geq P(q)$. Also, $S(q)=P(q)$ if and only if $S(q)$ is prime. (If $S(q)$ were prime but greater than $P(q)$, then since $q$ divides $S(q)$ !, it would also divide $(S(q)-1)$ ! , contradicting the minimality of $S(q)$.)

P. Erd'ós and I. Kastanas [9] observed that

$$
S(q)=P(q) \quad(\text { almost all } q)
$$

(Recall that a claim $C_{q}$ is true for almost all $q$ if the counting function $N(x)=\#\left\{q \leq x: C_{q}\right.$ is false $\}$ satisfies the asymptotic condition $N(x) / x \rightarrow 0$ as $x \rightarrow \infty$.) It follows that Theorem 1 implies an irrationality measure for $e$ involving the simpler function $P(q)$.

Corollary 1. For almost all q, the following inequality holds with any integer $p$ :

$$
\left|e-\frac{p}{q}\right|>\frac{1}{(P(q)+1) !} \text {. }
$$

When $q$ is a factorial, the statement is more definite.

Corollary 2. Fix $q=n !>1$. Then (12) holds for all $p$ if and only if $n$ is prime.

Proof. If $n$ is prime, then $P(q)=n$, so (4) and (9) imply (12) for all $p$. Conversely, if $n$ is composite, then $P(q)<n$, and (10) shows that (12) fails for certain $p$. 
Thus when $q>1$ is a factorial, (12) is true for all $p$ if and only if $S(q)=P(q)$. To illustrate this, take $\frac{p}{q}=\frac{65}{4 !}$ to be the left endpoint of $I_{4}$. Then $P(q)=3<4=S(q)$, and (12) does not hold, although of course (4) does:

$$
0.00833 \ldots=\frac{1}{5 !}<\left|e-\frac{65}{24}\right|=0.00994 \ldots<\frac{1}{4 !}=0.04166 \ldots
$$

5. A KNOWN IRRATIONALITY MEASURE FOR $e$. The following measure of irrationality for $e$ is well known: given any $\varepsilon>0$ there exists a positive constant $q(\varepsilon)$ such that

$$
\left|e-\frac{p}{q}\right|>\frac{1}{q^{2+\varepsilon}}
$$

for all $p$ and $q$ with $q \geq q(\varepsilon)$. This follows easily from the continued fraction expansion of $e$. (See, for example, [23]. For sharper inequalities than (13), see [3, Corollary 11.1], [4], [7], [10, pp. 112-113], and especially the elegant [26].)

Presumably, (13) is usually stronger than (4). We state this more precisely, and in a number-theoretic way that does not involve $e$.

Conjecture 1. The inequality $q^{2}<S(q)$ ! holds for almost all $q$. Equivalently, $q^{2}<P(q)$ ! for almost all $q$.

(The equivalence follows from (11).) This is no doubt true; the only thing lacking is a proof. (Compare [12], where A. Ivic proves an asymptotic formula for the counting function $N(x)=\#\{q \leq x: P(q)<S(q)\}$ and surveys earlier work, including [9].)

Conjecture 1 implies that (13) is almost always a better measure of irrationality for $e$ than those in Theorem 1 and Corollary 1. On the other hand, Theorem 1 applies to all $q>1$. Moreover, (4) is stronger than (13) for certain $q$. For example, let $q=n$ ! once more. Then (4) and (9) give (6), which is stronger than (13) if $n>2$, since

$$
(n+1) !<(n !)^{2} \quad(n \geq 3) .
$$

6. PARTIAL SUMS VS. CONVERGENTS. Theorem 1 yields other results on rational approximations to $e$ [24]. One is that for almost all $n$, the $n$-th partial sum $s_{n}$ of series (2) for $e$ is not a convergent to the simple continued fraction for $e$. Here $s_{0}=1$ and $s_{n}$ is the left endpoint of $I_{n}$ for $n \geq 1$. (In $1840 \mathrm{~J}$. Liouville [14] used the partial sums of the Taylor series for $e^{2}$ and $e^{-2}$ to prove that the equation $a e^{2}+b e^{-2}=c$ is impossible if $a$, $b$, and $c$ are integers with $a \neq 0$. In particular, $e^{4}$ is irrational.)

Let $q_{n}$ be the denominator of $s_{n}$ in lowest terms. When $q_{n}=n !$ (see [22, sequence A102470]), the result is more definite, and the proof is easy.

Corollary 3. If $q_{n}=n$ ! with $n \geq 3$, then $s_{n}$ cannot be a convergent to $e$. 
Proof. Use (4), (9), (14), and the fact that every convergent satisfies the reverse of inequality (13) with $\varepsilon=0[10$, p. 24], [17, p. 61].

When $q_{n}<n$ ! (for example, $q_{19}=19 ! / 4000$ - see [22, sequence A093101]), another argument is required, and we can only prove the assertion for almost all $n$. However, numerical evidence suggests that much more is true.

Conjecture 2. Only two partial sums of series (2) for e are convergents to e, namely, $s_{1}=2$ and $s_{3}=8 / 3$.

7. CANTOR'S THEOREM. A generalization of the construction in section 2 can be used to prove the following result of Cantor [5].

Theorem 2. Let $a_{0}, a_{1}, \ldots$ and $b_{1}, b_{2}, \ldots$ be integers satisfying the inequalities $b_{n} \geq 2$ and $0 \leq a_{n} \leq b_{n}-1$ for all $n \geq 1$. Assume that each prime divides infinitely many of the $b_{n}$. Then the sum of the convergent series

$$
a_{0}+\frac{a_{1}}{b_{1}}+\frac{a_{2}}{b_{1} b_{2}}+\frac{a_{3}}{b_{1} b_{2} b_{3}}+\cdots
$$

is irrational if and only if both $a_{n}>0$ and $a_{n}<b_{n}-1$ hold infinitely often.

For example, series (2) for $e$ and all subseries (such as $\Sigma_{n \geq 0} \frac{1}{(2 n) !}=\cosh 1$ and $\left.\Sigma_{n \geq 0} \frac{1}{(2 n+1) !}=\sinh 1\right)$ are irrational, but the sum $\Sigma_{n \geq 1} \frac{n-1}{n !}=1$ is rational.

An exposition of the "if" part of Cantor's theorem is given in [17, pp. 7-11]. For extensions of the theorem, see $[\mathbf{8}],[\mathbf{1 1}],[\mathbf{1 8}]$, and $[\mathbf{2 5}]$.

ADDENDUM. Here are some details on why the nested closed intervals $I_{n}$ constructed in section 2 have intersection $e$. Recall that $I_{1}=[2,3]$, and that for $n \geq 2$ we get $I_{n}$ from $I_{n-1}$ by cutting it into $n$ equal subintervals and taking the second one. The left-hand endpoints of $I_{1}, I_{2}, I_{3}, \ldots$ are $2,2+\frac{1}{2 !}, 2+\frac{1}{2 !}+\frac{1}{3 !}, \ldots$, which are also partial sums of the series (2) for $e$. Since the endpoints approach the intersection of the intervals, whose lengths tend to zero, the intersection is the single point $e$.

ACKNOWLEDGMENTS. Stefan Krämer pointed out the lack of geometric proofs of irrationality. The referee suggested a version of the question in section 2. Yann Bugeaud and Wadim Zudilin supplied references on the known irrationality measures for $e$. Aleksandar Ivic commented on Conjecture 1. Kyle Schalm did calculations [24] on Conjecture 2, and Yuri Nesterenko related it to Liouville's proof. I am grateful to them all. 


\section{REFERENCES}

1. M. Aigner and G. Ziegler, Proofs from THE BOOK, 2nd. ed., Springer-Verlag, New York, 2001.

2. T. Apostol, Irrationality of the square root of two - a geometric proof, Amer. Math. Monthly 107 (2000) 841-842.

3. J. Borwein and P. Borwein, Pi and the AGM: A Study in Analytic Number Theory and Computational Complexity, John Wiley \& Sons, New York, 1987.

4. P. Bundschuh, Irrationalitätsmasse für $e^{a}, a \neq 0$ rational oder Liouville-Zahl, Math. Ann. 192 (1971) 229-242.

5. G. Cantor, Über die einfachen Zahlensysteme, Z. Math. und Phys. 14 (1869) 121-128; also in Collected Works, Springer-Verlag, Berlin, 1932, pp. 35-42.

6. J. L. Coolidge, The number e, Amer. Math. Monthly 57 (1950) 591-602.

7. C. S. Davis, Rational approximations to e, J. Austral. Math. Soc. Ser. A 25 (1978) 497-502.

8. P. H. Diananda and A. Oppenheim, Criteria for irrationality of certain classes of numbers. II, Amer. Math. Monthly 62 (1955) 222-225.

9. P. Erdo's and I. Kastanas, Problem/Solution 6674: The smallest factorial that is a multiple of n, Amer. Math. Monthly 101 (1994) 179.

10. N. I. Fel'dman and Yu. V. Nesterenko, Transcendental Numbers, Encyclopaedia of Mathematical Sciences, A. N. Parshin and I. R. Shafarevich (Eds.), Vol. 44: Number Theory IV, Springer-Verlag, New York, 1998.

11. J. Hancl and R. Tijdeman, On the irrationality of Cantor series, J. Reine Angew. Math. 571 (2004) 145-158.

12. A. Ivić, On a problem of Erdös involving the largest prime factor of $n$, Monatsh. Math. 145 (2005) 35-46.

13. A. J. Kempner, Concerning the smallest integer $m$ ! divisible by a given integer $n$, Amer. Math. Monthly 25 (1918) 204-210.

14. J. Liouville, Addition à la note sur l'irrationalité du nombre e, J. Math. Pures Appl. 5 (1840) 193-194.

15. E. Maor, e: The Story of a Number, Princeton University Press, Princeton, 1994.

16. J. A. Nathan, The irrationality of $e^{x}$ for nonzero rational x, Amer. Math. Monthly 105 (1998) 762-763.

17. I. Niven, Irrational Numbers, Carus Math. Monographs, no. 11, Mathematical Association of America, distributed by John Wiley \& Sons, New York, 1956.

18. A. Oppenheim, Criteria for irrationality of certain classes of numbers, Amer. Math. Monthly 61 (1954) 235-241.

19. A. E. Parks, $\pi, e$, and other irrational numbers, Amer. Math. Monthly 93 (1986) $722-$ 723.

20. L. L. Pennisi, Elementary proof that $e$ is irrational, Amer. Math. Monthly 60 (1953) 474.

21. P. Ribenboim, My Numbers, My Friends, Springer-Verlag, New York, 2000.

22. N. J. A. Sloane, The On-Line Encyclopedia of Integer Sequences (2005), published electronically at http://www .research.att.com/ njas/sequences/.

23. J. Sondow, Irrationality measures, irrationality bases, and a theorem of Jarník (2004, preprint); available at http://arXiv.org/abs/math/0406300. 
24. __ Which partial sums of the Taylor series for $e$ are convergents to $e$ ?, with an Appendix by K. Schalm (2006, preprint); available at http://arXiv.org/find/math/1/au:+sondow/0/1/0/all.

25. M. R. Spiegel, On a class of irrational numbers, Amer. Math. Monthly 60 (1953) 2728.

26. B. G. Tasoev, Rational approximations to certain numbers, Math. Notes 67 (2000) 786-791.

27. B. Turner, A geometric proof that $\sqrt{ } 2$ is irrational, Math. Mag. 50 (1977) 263.

28. E. W. Weisstein et al, Smarandache function, MathWorld-A Wolfram Web Resource, published electronically at http://mathworld.wolfram.com/SmarandacheFunction.html.

209 West 97th Street, New York, NY 10025

jsondow@alumni.princeton.edu 